\title{
MULTIPLIERS ON THE SPACE OF SEMIPERIODIC SEQUENCES
}

\author{
$\mathrm{BY}$ \\ MANUEL NÚÑEZ JIMÉNEZ
}

\begin{abstract}
Semiperiodic sequences are defined to be the uniform limit of periodic sequences. They form a space of continuous functions on a compact group $\Delta$. We study the properties of the Radon measures on $\Delta$ in order to classify the multipliers for the space of semiperiodic sequences, paying special attention to those which can be realized as transference functions of physically constructible filters.
\end{abstract}

Introduction. Almost periodic functions have been the subject of active research since their definition in 1925 by Harald Bohr. Their main interest lies in the fact that they form the smallest Banach space containing all the periodic functions of one real variable, thus becoming the natural background for the study of linear problems involving periodic functions. Subsequently the definition was extended in the following form: a function defined on a locally compact group is almost periodic if it is the uniform limit of a sequence of trigonometric polynomials. In particular, almost periodic sequences correspond to the group $\mathbf{Z}$ of integers.

Sequences are in some respects a better model than functions for the study of periodic processes. The set $p$ of all the complex-valued periodic sequences, endowed with the usual componentwise sum and product by scalars, is a normed vector space for the supremum norm given by

$$
\left\|\left(x_{n}\right)_{n \in \mathbf{Z}}\right\|_{\infty}=\sup _{n \in \mathbf{Z}}\left|x_{n}\right| .
$$

The completion of $p$ does not coincide with the set of almost periodic sequences. Instead, it is a new space sp, which will be called the space of semiperiodic sequences.

An element of sp is easily seen as a sequence $\left(x_{n}\right)_{n \in \mathbf{Z}}$ with the property that for every $\varepsilon>0$, there exists a "semiperiod" $T$ such that for every $m, n \in \mathbf{Z}$ we have $\left|x_{m+n T}-x_{m}\right|<\varepsilon$. Semiperiodic nonperiodic sequences usually arise when we study limits of periodic sequences with unbounded periods. For instance, take

$$
\begin{aligned}
& e_{1}=(\ldots, 1,0,1,0,1,0,1, \ldots), \\
& e_{2}=(\ldots, 1,0,0,1,0,0,1, \ldots), \\
& e_{3}=(\ldots, 1,0,0,0,1,0,0, \ldots), \\
& e_{4}=(\ldots, 1,0,0,0,0,1,0, \ldots), \ldots
\end{aligned}
$$

Then $\sum_{k=1}^{\infty} e_{k} / 2^{k}$ is not periodic, since the zero does not appear more than twice in the sequence.

Received by the editors February 22, 1984 and, in revised form, October 16, 1984.

1980 Mathematics Subject Classification. Primary 22B10, 43A25, 30A76, 42A18, 28 A30.

Key words and phrases. Semiperiodic sequences, Radon measures, Fourier analysis, multipliers. 
There is a characterization of almost periodic sequences (see [1] ) which is similar to the above one, but it differs in the fact that the set of almost periods does not need to be equidistributed, but only a relatively dense set.

Our main objective in this paper is the classification of filters of semiperiodic signals. Recall that a filter is a device which acts on a given set of signals separately on each frequency present in them; mathematically speaking, it is a linear operator

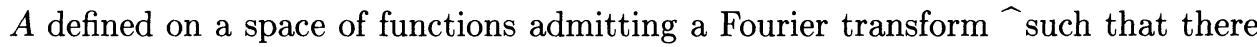
exists a function $g$ (a multiplier) with the property that we have $(A f)^{\widehat{ }}=g \hat{f}$ for all $f$. The multiplier $g$ is also called the transference function of the filter.

A classical characterization of multipliers relates filters on semiperiodic signals with the representation of sp as a space of continuous functions defined on a compact group $\Delta$. In fact, multipliers are the Fourier transforms of Radon measures on $\Delta$. In order to obtain a classification of these measures, we prove the following results.

LEMMA A. The elements in the dual space of $\mathrm{sp}$ are doubly infinite matrices of complex numbers

$$
\left[\begin{array}{cccc}
u_{1}^{1} & 0 & 0 & \cdots \\
u_{1}^{2} & u_{2}^{2} & 0 & \cdots \\
u_{1}^{3} & u_{2}^{3} & u_{3}^{3} & \cdots \\
\cdots & \cdots & \cdots & \cdots
\end{array}\right]
$$

verifying the compatibility condition

$$
u_{i}^{T}=u_{i}^{k T}+u_{i+T}^{k T}+\cdots+u_{i+(k-1) T}^{k T}
$$

and $\sup _{T} \sum_{i=1}^{T}\left|u_{i}^{T}\right|<\infty$.

From this lemma immediately follows

THEOREM B. The space sp can be identified with the space of complex-valued continuous functions defined on the compact group $\Delta=\varliminf_{T \in \mathbf{N}}(\mathbf{Z} / T \mathbf{Z})$ (projective limit relative to the trivial projections $\mathbf{Z} / k T \mathbf{Z} \rightarrow \mathbf{Z} / T \mathbf{Z}$ ).

The following theorem relates the description of continuous functionals on sp in Lemma $\mathrm{A}$ with the Radon measures on $\Delta$, which constitute $\mathrm{sp}^{\prime}$ according to the theorem of Riesz:

THEOREM C. Let $\left(u_{i}^{j}\right)$ be a nonnegative matrix in $\mathrm{sp}^{\prime}$. For $w \in \Delta$, denote by $w_{T}$ its projections on $\mathbf{Z} / T \mathbf{Z}$. Let $T_{1}, \ldots, T_{n}, \ldots$ be a sequence of integers such that $T_{k}$ divides $T_{k+1}$ and such that for each $S \in \mathbf{N}$ one has $S \mid T_{n}$ for some $n$ (i.e. increasing and cofinal for the order relation of divisibility on $\mathbf{N}$ ). Set

$$
\begin{aligned}
& A_{1}=\left\{w \in \Delta \mid \exists \lim _{n \rightarrow \infty} T_{n} u_{w_{T_{n}}}^{T_{n}}(\text { denoted by } h(w))\right\}, \\
& A_{2}=\left\{w \in \Delta \mid \lim _{n \rightarrow \infty} T_{n} u_{w_{T_{n}}}^{T_{n}}=\infty\right\}, \\
& B_{1}=\left\{w \in A_{2} \mid \lim _{n \rightarrow \infty} u_{w_{T_{n}}}^{T_{n}}=0\right\}, \\
& B_{2}=\left\{w \in A_{2} \mid \lim _{n \rightarrow \infty} u_{w_{T_{n}}}^{T_{n}}=g(w) \neq 0\right\}
\end{aligned}
$$

(the limits in the definitions of $B_{1}$ and $B_{2}$ exist because $\left(u_{w_{T_{n}}}^{T_{n}}\right)$ is a decreasing sequence). Then, denoting by $m$ the Haar measure on $\Delta$, one has the following. 
(1) $m\left(\Delta-A_{1}\right)=0$.

(2) The $m$-almost everywhere defined function $h$ represents the component of $\left(u_{i}^{j}\right)$ which is a Radon measure on $\Delta$ absolutely continuous with respect to $m$. Moreover, this component $\left(v_{i}^{j}\right)$ is given by

$$
v_{i}^{j}=\int_{\Delta_{i}^{j} \cap A_{1}} h(w) d m(w)
$$

$\left(\Delta_{i}^{j}=\left\{w \in \Delta \mid w_{j}=i\right\}\right)$.

(3) The matrix $\left(u_{i}^{j}-v_{i}^{j}\right)$ represents a measure $\mu_{s}$ which is singular to $m$, and verifies $\mu_{s}\left(\Delta-A_{2}\right)=0$.

(4) $B_{2}$ is the set of points on which the atomic component $\nu$ of $\mu_{s}$ is concentrated. It is a countable set, and the matrix $\left(z_{i}^{j}\right)$ associated to $\nu$ is given by

$$
z_{i}^{j}=\sum_{w \in B_{2} \cap \Delta_{i}^{j}} g(w) .
$$

Moreover, $\nu\left(\Delta-B_{2}\right)=0$.

(5) $B_{1}$ is the set of points on which the diffuse component of $\mu_{s}$ is concentrated. Its associated matrix is given by $\left(u_{i}^{j}-v_{i}^{j}+z_{i}^{j}\right)$.

The proof of this long theorem involves most of $\S 1$. In $\S 2$ we study the Fourier analysis on $\Delta$. Its dual group can be identified to the discrete set $G$ of all the complex roots of the unity. The aforesaid characterization of multipliers, due to Helson and Edwards (see $[\mathbf{3}, \mathbf{6}]$ ), provides a first result: recalling that the weight of a polynomial $P(z)=\sum a_{n} z^{n}$ is defined by $\operatorname{wt}(P)=\sum\left|a_{n}\right|$, we obtain

THEOREM D. A function $f: G \rightarrow \mathbf{C}$ is a multiplier if and only if it is the pointwise limit of a sequence of polynomials of bounded weight.

By reasons of empirical nature, one would expect that a multiplier associated to a really constructible filter of signals would keep some regularity on its effects on the different frequencies. The classification in Theorem $\mathrm{C}$ proves that this assumption drastically reduces the form of the multiplier; we obtain

THEOREM E. If a multiplier of $\mathrm{sp}$ is an absolutely continuous function on some subinterval of $G$, then it takes the form $g(z)=\sum_{n=0}^{\infty} a_{n} z^{n}$ on this subinterval, where $\sum\left|a_{n}\right|<\infty$; the analytic function $g$ depends only on the multiplier, not on the subinterval.

In particular, phase-shift filters (i.e. multipliers of modulus one) have a very simple form:

THEOREM F. Every multiplier which is a uniformly continuous function for the usual topology of $G$ and has modulus 1 is a Blaschke product of type

$$
g(z)=v \prod_{j=1}^{p} \frac{z-a_{j}}{1-\bar{a}_{j} z},
$$

where $a_{1}, \ldots, a_{p}$ belong to the disk $\{z|| z \mid<1\}$ and $|v|=1$.

Therefore the action of such a filter is determined by its effect on a finite set of frequencies. 


\section{Representations of $\mathrm{sp}^{\prime}$.}

PROOF OF LEMMA A. Each continuous functional $u$ on the finite-dimensional subspace of all the $T$-periodic sequences must be given by $T$ complex numbers $u_{i}^{T}$, i.e. one has

$$
\langle u, x\rangle=\sum_{i=1}^{T} u_{i}^{T} x_{i}
$$

for each $T$-periodic sequence $x=\left(x_{n}\right)_{n \in \mathbf{Z}}$. Since a $T$-periodic sequence is also $k T$-periodic one has

$$
\sum_{i=1}^{T} u_{i}^{T} x_{i}=\sum_{i=1}^{k T} u_{i}^{k T} x_{i}
$$

which implies the relation of compatibility. On the other hand, the norm of this restricted functional is $\sum_{i=1}^{T}\left|u_{i}^{T}\right|$; thus $\sup _{T} \sum_{i=1}^{T}\left|u_{i}^{T}\right|$ is the norm of the mapping taken in the dense subspace of periodic sequences, hence in the whole space sp. The action of such a functional on a nonperiodic sequence is given by a limit.

ProOF OF THEOREM B. Componentwise product makes sp a $B^{*}$-algebra. Therefore we will study the multiplicative functionals on $p$ (hence on $\mathrm{sp}$ ) in order to find the Gelfand representation of sp as a space of continuous functions. Since the only multiplicative functionals on $\mathbf{C}^{T}$ are the projections, every row $\left(u_{1}^{T}, \ldots, u_{T}^{T}\right)$ must have at most one element 1 . It is not hard to see that if the functional is not null then there exist on this row exactly one element 1 and $T-1$ elements equal to 0 .

Let $r_{T}$ denote the place on which the element 1 is located in the $T$ th row. The compatibility relations state that $r_{n T}+T \mathbf{Z}=r_{T}+T \mathbf{Z}$, namely $\left(r_{T}\right)_{T \in \mathbf{N}}$ is an element of $\lim (\mathbf{Z} / T \mathbf{Z})=\Delta$. It is easy to see that the Gelfand (i.e. weak) topology on $\Delta$ is the restriction to $\Delta$ of the product topology in $\prod_{T}(\mathbf{Z} / T \mathbf{Z})(\mathbf{Z} / T \mathbf{Z}$ discrete $)$, namely the projective limit of the discrete topologies. Thus Gelfand theory proves our result.

At this point a more specific representation of the isomorphism between sp and $C(\Delta)$ is desirable. The Gelfand transform assigns to every semiperiodic sequence $x$ the function $\tilde{x}$, defined on the dense set $\tilde{\mathbf{Z}}=\left\{(j+T \mathbf{Z})_{T \in \mathbf{N}} \mid j \in \mathbf{Z}\right\}$ by the formula $\tilde{x}\left((j+T \mathbf{Z})_{T \in \mathbf{N}}\right)=x_{j}$. Hence the action of $\tilde{x}$ on a point $w=\left(w_{T}\right)_{T \in \mathbf{N}} \in \Delta$ is given by $\tilde{x}(w)=\lim _{j \in \mathbf{N}} x_{j}$, where the limit of this net follows the order relation of divisibility in $\mathbf{N}$. We see that the function $\tilde{x}$ is obtained by adding to $x$ all its possible values of adherence following subsequences of increasing (in the sense of divisibility) index.

For instance, the Gelfand transform of a $T$-periodic sequence $\left(x_{n}\right)$ is the step function $\sum_{j=1}^{T} x_{j} \kappa_{\Delta_{j}^{T}}$, where $\Delta_{j}^{T}$ is defined in Theorem C.

One could visualize $\Delta$ as a set of descending columns of 1's (located according to the compatibility relation). Then $T$-periodic sequences are the functions whose action on a given column only depends on the first $T$ steps of it.

By using the well-known algebraic result which asserts that $\Delta$ can be identified to the product of the rings of $p$-adic integers $\mathbf{Z}_{p}$ when $p$ ranges over the set of prime numbers, we can get some information about the topological properties of sp. Recall that $\mathbf{Z}_{p}$ is a compact, perfect and ultrametric space, so the same happens to $\Delta$. 
COROLLARY 1.1. For every compact set of $\mathbf{C}$, there exists a semiperiodic sequence whose range is dense in it.

PROOF. We have seen that the range of a sequence $x$ is the set $\tilde{x}(\tilde{\mathbf{Z}})$, where $\tilde{\mathbf{Z}}$ is dense in $\Delta$, whence $\tilde{x}(\tilde{\mathbf{Z}})$ is dense in $\tilde{x}(\Delta)$. Since $\Delta$ is perfect, it is homeomorphic to the Cantor set, which can be mapped onto every compact subset of $\mathbf{C}$.

Our next corollary is a consequence of

THEOREM (MILUTIN (1966)). If $X$ is an uncountable, metrizable and compact space, then $C(X)=C([0,1])$ (see for example $[\mathbf{4}])$.

COROLLARY 1.2. sp is isomorphic to the normed space of continuous functions on $[0,1]$.

The fact that sp is $C(\Delta)$ poses the following questions: How are measures in $\Delta$ and matrices in $\mathrm{sp}^{\prime}$ related? Let $x$ be a $T$-periodic sequence. The action of a continuous functional on $x$ is given by $\int_{\Delta} \tilde{x}(u) d \mu(u)$ for some Radon measure $\mu$, or by $\sum_{j=1}^{T} u_{j}^{T} x_{j}$.

Since the expression of $\tilde{x}$ is $\sum_{j=1}^{T} x_{j} \kappa_{\Delta_{j}^{T}}$, we obtain $u_{j}^{T}=\mu\left(\Delta_{j}^{T}\right)$. Hence the matrix associated to the functional is given by the measure of a class of subsets of $\Delta$; those coming from finite partitions in $T$ "equal" parts. $\left(\Delta_{j}^{T}\right.$ is the set of columns of 1 's having the 1 in the $T$ th row located in the $j$ th place.) As an important example, let us take the Haar measure of mass 1 on $\Delta$. Since $\Delta_{j}^{T}$ can be obtained by adding $(j-k+T \mathbf{Z})_{T \in \mathbf{N}}$ to $\Delta_{k}^{T}$, they must have the same measure, so $m\left(\Delta_{j}^{T}\right)=1 / T$. Hence the functional associated to $m$ is the mean value

$$
x \rightarrow \lim _{T \rightarrow \infty}\left(\frac{1}{T} \sum_{k=1}^{T} x_{k}\right) .
$$

Our next aim is to give a characterization of the properties of a measure in terms of the entries of its associated matrix. We will use some classical theorems about derivations of measures, following the terminology of $[\mathbf{5}]$.

LEMMA 1.3. Let $A$ be a sequence of integers $T_{1}, \ldots, T_{n}, \ldots$ increasing and cofinal for the divisibility relation. Set $H_{T}=\left\{\Delta_{1}^{T}, \ldots, \Delta_{T}^{T}\right\}$. Then $H=\left(H_{T}\right)_{T \in A}$ is a monotone sequence of nets, and $\bigcup_{T \in A} H_{T}$ is a basis for the topology of $\Delta$.

Proof. First, note that if $S \mid T$ and $u \in \Delta_{i}^{S} \cap \Delta_{j}^{T} \neq \varnothing$, then $u_{S}=i, u_{T}=j$; hence $j-i \in S \mathbf{Z}$ and every element whose image in $\mathbf{Z} / T \mathbf{Z}$ is $j$ must have $i$ as its image in $\mathbf{Z} / S \mathbf{Z}$.

Therefore $\left(H_{T}\right)_{T \in A}$ is a monotone sequence of nets. On the other hand, a basis for the topology of $\Delta$ is formed by the sets $\left(\Delta_{i}^{T}\right)_{i=1, \ldots, T: T \in \mathbf{N}}$. For every $u \in \Delta$ and every neighborhood $\Delta_{u_{T}}^{T}$ of $u$, there exists an $S \in A$ such that $T \mid S$, hence $u \in \Delta_{u_{S}}^{S} \subset \Delta_{u_{T}}^{T}$.

LEMMA 1.4. The monotone sequence of nets $\left(H_{T}\right)_{T \in A}$ is regular with respect to $m$ on each measurable set.

Proof. Since $m$ is a Radon measure, it is regular. Thus for $\varepsilon>0$ and a measurable set $E$ there exists an open set $V$ such that $E \subset V, m(V-E)<\varepsilon$. The result follows from the fact that $V$ is a union of sets of the above countable basis. 
LEMMA 1.5. Let $W$ be a set which is the union of open sets of the form $\Delta_{i}^{T}$, where $i \leq T$ and $T \in A$. If $t<m(W)$, then there exist finitely many of these open sets such that they are pairwise disjoint and the sum of their measures is greater than $t$.

PROOF. Since $m$ is a regular measure, there exists a compact subset $K$ of $W$ with $m(K)>t$. Since $W$ is a union of open sets, $K$ can be covered by a finite subclass of these sets. For any two different ones of them, $\Delta_{i}^{T}, \Delta_{j}^{S}$, one has either $T \mid S$ or $S \mid T$, so either they are disjoint or one of them is contained in the other. If the second case occurs, the smallest one can be removed. The resulting subclass fulfills the required properties.

LEMMA 1.6. Let $\mu$ be a nonnegative Radon measure on $\Delta$ and $B$ a Borel set such that $\mu(B)=0$. Then there exists $B^{\prime} \subset B$ such that

(1) $m\left(B-B^{\prime}\right)=0$,

(2) $\forall u \in B^{\prime}, \lim _{T \in A, T \rightarrow \infty} T \mu\left(\Delta_{u_{T}}^{T}\right)=0$.

Proof. Let $B^{\prime}$ be the set of those $u \in B$ verifying (2). (Note that $\mu\left(\Delta_{u_{T}}^{T}\right)$ is precisely the $u_{T}$ th entry of the $T$ th row of the matrix representing $\mu$.) We will check that $m\left(B-B^{\prime}\right)=0$.

Set $P_{j}=\left\{u \in B \mid \varlimsup_{T \in A, T \rightarrow \infty} T \mu\left(\Delta_{u_{T}}^{T}\right)>1 / j\right\}$. Then one has $B-B^{\prime}=\bigcup_{j} P_{j}$, so it suffices to see that $m\left(P_{j}\right)=0$. Take $\varepsilon>0$; there exists an open set $V$ with $B \subset V$ and $\mu(V)<\varepsilon$.

For $u \in P_{j}$, take $T \in A$ with $\Delta_{u_{T}}^{T} \subset V$ and

$$
m\left(\Delta_{u_{T}}^{T}\right)=\frac{1}{T}<j \mu\left(\Delta_{u_{T}}^{T}\right)
$$

and let $W$ be the union of the sets $\Delta_{u_{T}}^{T}$. By Lemma 1.5, there exists a finite subclass of pairwise disjoint sets $\Delta_{u_{T_{i}}}^{T_{i}}$ for $t<m(W)$ and $i=1, \ldots, n$, for which

$$
t<\sum_{i=1}^{n} m\left(\Delta_{u_{T_{i}}}^{T_{i}}\right)<j \sum_{i=1}^{n} \mu\left(\Delta_{u_{T_{i}}}^{T_{i}}\right)=j \mu\left(\bigcup_{i=1}^{n} \Delta_{u_{T_{i}}}^{T_{i}}\right) \leq j \mu(V)<j \varepsilon .
$$

Therefore $m(W)<j \varepsilon$, whence $m\left(P_{j}\right) \leq j \varepsilon \forall \varepsilon>0$, so $m\left(P_{j}\right)=0$ as required.

PROPOSITION 1.7. Let $\mu$ be a complex Radon measure on $\Delta$ and $A$ a sequence of integers as in Lemma 1.3. Then one has:

(1) The limit $D_{H} \mu(u)=\lim _{T \in A, T \rightarrow \infty} T \mu\left(\Delta_{u_{T}}^{T}\right)$ exists m-a.e. on $\Delta$.

(2) $D_{H} \mu \in L^{1}(\Delta, m)$.

(3) For every Borel set $E$ one has

$$
\mu(E)=\mu_{s}(E)+\int_{E} D_{H} \mu(u) d m(u)
$$

where $\mu_{s} \perp m$ and $D_{H} \mu_{s}=0, m$-a.e.

(Therefore the above is the Lebesgue decomposition of $\mu$.)

ProOF. Let $\mu=\mu_{s}+\nu$, where $\mu_{s} \perp m$ and $\nu \ll m$ is the Lebesgue decomposition of $\mu$. All the measures $\mu, \mu_{s}, \nu$ are regular. We must see that $D_{H} \mu(u)=\lim _{T \in A} T \mu\left(\Delta_{u_{T}}^{T}\right)$ coincides with the Radon-Nikodým derivative $h=$ $d \nu / d m$. Consider the Jordan decomposition of $\mu_{s}$ as a sum of nonnegative measures (by the Hahn decomposition theorem each of them and $m$ are singular). By 
applying Lemma 1.6 to each of these measures, we get $D_{H} \mu_{s}=0 m$-a.e., whence $D_{H} \mu=D_{H} \nu m$-a.e.

Now Theorem 36.4 in $[\mathbf{5}]$ shows that $D_{H} \nu=h$ a.e.

Note that the identification of $D_{H} \nu$ with the derivative of Radon-Nikodým proves that the limit does not depend on the particular choice of the sequence $A$.

PROPOSITION 1.8. Let $\mu$ be a nonnegative Radon measure on $\Delta$ which is singular to $m$. Then

$$
D_{H} \mu(u)=\lim _{\substack{T \in A \\ T \rightarrow \infty}} T \mu\left(\Delta_{u_{T}}^{T}\right)=\infty \quad \mu \text {-a.e. }
$$

ProOF. Let $E_{j}$ be the set of those $u \in \Delta$ for which there exists a subsequence $A(u) \subset A$ such that $T \mu\left(\Delta_{u_{T}}^{T}\right)<j$ for all $T \in A(u)$. We will prove that the $\mu$ measure of the set $\bigcup_{j} E_{j}$ is zero. Since $\mu$ is singular to $m, \mu$ vanishes outside an open set $V$ with $m(V)<\varepsilon$ for $\varepsilon>0$. For $u \in V \cap E_{j}$, there exists $T \in A(u)$ such that $\Delta_{u_{T}}^{T} \subset V$. Take $T$ to be the minimum element in $A(u)$ with this property. If two of these sets $\Delta_{u_{T}}^{T}$ and $\Delta_{u_{S}}^{S}$ meet, then one of them must be contained in the other.

Removing all the redundant sets, we get $V \cap E_{j} \subset \bigcup_{i} \Delta_{u_{T_{i}}}^{T_{i}}$, where the sets of the union at the right-hand side member are pairwise disjoint, all of them verifying

$$
\mu\left(\Delta_{u_{T_{i}}}^{T_{i}}\right)<j m\left(\Delta_{u_{T_{i}}}^{T_{i}}\right)
$$

and they form a countable class. So one has

$$
\begin{aligned}
\mu\left(E_{j}\right) & =\mu\left(V \cap E_{j}\right) \leq \mu\left(\bigcup_{i} \Delta_{u_{T_{i}}}^{T_{i}}\right)=\sum_{i} \mu\left(\Delta_{u_{T_{i}}}^{T_{i}}\right)<j \sum_{i} m\left(\Delta_{u_{T_{i}}}^{T_{i}}\right) \\
& =j m\left(\bigcup_{i} \Delta_{u_{T_{i}}}^{T_{i}}\right) \leq j m(V)<j \varepsilon
\end{aligned}
$$

and hence $\mu\left(E_{j}\right)=0$, whence $\mu\left(\bigcup_{j} E_{j}\right)=0$.

REMARK. The result in this proposition is stronger than the analogous one for the Lebesgue measure. This is due to the fact that every union of sets of the form $\Delta_{i}^{T}$ becomes, after removing some of them, a union of pairwise disjoint sets.

Proposition 1.9. Let $\mu$ be a diffuse measure. Then the net $\left(\mu\left(\Delta_{u_{T}}^{T}\right)\right)_{T \in \mathbf{N}}$ (following the relation of divisibility) converges uniformly to 0 in $\Delta$.

PROOF. It is enough to prove the proposition for nonnegative measures, as the Jordan components of a diffuse measure are also diffuse. Assume that the statement is false for a nonnegative $\mu$. We may construct a sequence $T_{1}, \ldots, T_{n}, \ldots$ with $T_{k} \mid T_{k+1}$ such that $\mu\left(\Delta_{u_{T_{n}}}^{T_{n}}\right)$ does not converge uniformly on $\Delta$. Consider the functions

$$
g_{n}=\sum_{h=1}^{T_{n}} \mu\left(\Delta_{h}^{T_{n}}\right) \kappa_{\Delta_{h}}^{T_{n}} .
$$

The compatibility relations and the fact that $\mu \geq 0$ imply that $\left(g_{n}\right)$ is a decreasing sequence of functions. Its limit is $\lim _{n \rightarrow \infty} \mu\left(\Delta_{u_{T_{n}}}^{T_{n}}\right)$, which is zero since $\mu$ is diffuse. Now, by Dini's theorem, the convergence is uniform, which is a contradiction. This completes the proof of the proposition. 
Collecting the above results together, the proof of Theorem $\mathrm{C}$ follows. This theorem is useful for the classification of functionals in $\mathrm{sp}^{\prime}$, once one has made the Jordan decomposition in order to handle nonnegative matrices.

Note that an atomic measure on $\Delta$ subordinated to $\tilde{\mathbf{Z}}$ represents a functional in $\mathrm{sp}^{\prime}$ if and only if it is of type $\left(x_{n}\right) \rightarrow \sum_{n=1}^{\infty} a_{n} x_{n}$ with $\sum_{n}\left|a_{n}\right|<\infty\left(a_{n}\right.$ is the measure of the element $\left.(n+T \mathbf{Z})_{T \in \mathbf{N}} \in \Delta\right)$.

2. Harmonic analysis and multipliers. The dual group of $\Delta$ is the discrete group $G$ of all the roots of unity (see [2]). This follows from the fact that the dual of $\mathbf{Z} / T \mathbf{Z}$ is the group $G_{T}$ of the $T$ th roots of unity, where the action of $z \in G_{T}$ on $r+T \mathbf{Z}$ is given by the power $z^{r}$.

At this point a comparison with almost periodic sequences can be illustrative. Let $H$ be a locally compact group, $\hat{H}_{d}$ its dual endowed with the discrete topology and $b H$ the dual of this new group. $b H$ is called the Bohr compactification of $H$, and a classical theorem asserts that the set of almost periodic functions on $H$ can be identified as $C(b H)$. In our case, $H=\mathbf{Z}, \hat{H}=\mathbf{S}$ (the unit torus) and $\hat{\mathbf{S}}_{d}=b \mathbf{Z}$; on the other hand, by the theorem of duality of Pontryagin, $\hat{G}=\Delta$. The Bohr compactification $b \mathbf{Z}$ can be represented (see [2, p. 405]) as $\Sigma_{a}^{c} \times \Delta$, where $\Sigma_{a}$ (the $a$-adic solenoid) corresponds to the elements of $\mathbf{S}$ which are not roots of the unity, and $c$ (the power of the continuum) is the dimension of $\mathbf{R}$ on $\mathbf{Q}$. This reflects the fact that an almost periodic sequence is the uniform limit of trigonometric polynomials $\left(\sum_{z} a_{z} z^{n}\right)_{n \in \mathbf{Z}},|z|=1$, while for a semiperiodic one the $z$ 's appearing in the polynomials must be roots of unity.

The Fourier series associated to a $T$-periodic sequence is merely its expression as a trigonometric polynomial, all Fourier coefficients vanishing outside $G_{T}$. On the other hand, the Fourier transform of a measure $\mu=\left(u_{i}^{T}\right)$ at a point $z \in G_{T}$ is given by

$$
\hat{\mu}(z)=\int_{\Delta} z^{-r} d \mu(r)=\sum_{j=1}^{T} z^{-j} \mu\left(\Delta_{j}^{T}\right)=\sum_{j=1}^{T} u_{j}^{T} z^{-j}
$$

We will study the multipliers in sp, i.e. those functions $f: G \rightarrow \mathbf{C}$ for which there exists a continuous linear operator $A: \mathrm{sp} \rightarrow$ sp verifying $(A x)^{\widehat{ }}=f \hat{x}$, where - denotes the Fourier transform.

For such an $A$, the adjoint operator ${ }^{t} A$ defined on the space $M(\Delta)$ of the Radon measures over $\Delta$ verifies a similar condition and maps $L^{1}(\Delta) \subset M(\Delta)$ into $M(\Delta)$. A classical theorem by Helson and Edwards (see [6]) asserts that ${ }^{t} A(f)=\mu * f$ for some $\mu \in M(\Delta)$. Therefore the multipliers, or transference functions, are precisely the Fourier transforms of Radon measures. An intrinsic characterization of them is given by Theorem $\mathrm{D}$.

PROOF OF THEOREM D. Let $f$ be a multiplier and $\mu$ its associated measure. Then one has

$$
\hat{f}(z)=\hat{\mu}(z)=\sum_{k=1}^{T} u_{k}^{T} z^{-k}=\sum_{k=1}^{T} u_{T-k}^{T} z^{k} \quad \forall z \in G_{T}
$$


Now, let $T_{1}, \ldots, T_{n}, \ldots$ be a sequence of integers increasing and cofinal for the relation of divisibility. Set

$$
p_{n}(z)=\sum_{k=1}^{T_{n}} u_{T_{n}-k}^{T_{n}} z^{k} .
$$

One has $p_{n}(z)=\hat{\mu}(z) \forall z \in G_{T_{n}}$, so for all $z \in G$ the sequence $p_{n}(z)$ converges to $f(z)$. Moreover,

$$
\operatorname{wt}\left(p_{n}\right)=\sum_{k=1}^{T_{n}}\left|u_{T_{n}-k}^{T_{n}}\right| \leq\|\mu\|
$$

so $f$ is the pointwise limit of a sequence of polynomials of bounded weight.

Conversely, let $f$ be the pointwise limit of $\left(p_{n}\right), \operatorname{wt}\left(p_{n}\right)<M$. Consider the operators $p_{n}(S)$, where $S$ is the shift operator given by $S\left(\left(x_{n}\right)_{n \in \mathbf{Z}}\right)=\left(x_{n+1}\right)_{n \in \mathbf{Z}}$.

Since $\left\|p_{n}(S)\right\| \leq \operatorname{wt}\left(p_{n}\right) \leq M$, the operators form an equicontinuous set, so the convergence in a total set implies (and in fact is equivalent to) precompact convergence. The action of $S$ on the $T$-periodic sequence of the powers of a $T$ th root of unity $z$ is the product of $z$ and this sequence, namely

$$
\left(p_{n}(S) x\right)^{\uparrow}(z)=p_{n}(z) \hat{x}(z),
$$

which converges to the function $f \hat{x}$.

Since $p_{n}(S) x$ converges for every periodic sequence $x$, the sequence $p_{n}(S)$ converges to some continuous operator $A$ on sp. By Lebesgue's theorem, one has

$$
(A(x) \hat{)}(z)=f(z) \hat{x}(z) .
$$

Therefore $f$ is a multiplier.

Joining this result with the general theorem about idempotent measures $[6, p$. $60]$ one obtains the following

COROLLARY 2.1. A subset belongs to the smallest $\sigma$-algebra containing all the cosets of $G$ (i.e. all the classes modulo subgroups) if and only if its characteristic function is the pointwise limit of a sequence of polynomials with bounded weight.

Now we will study multipliers as transference functions of physically constructible filters. Although theoretically possible, a real filter cannot suppress a determined frequency leaving all the others unmodified, no matter how close to the first one. We will see that even the weakest requirement on regularity for the usual topology of $G$ implies that the multiplier is an analytic function, the Fourier transform of an atomic measure.

Lemma 2.2. Let $f$ be a multiplier. Assume that $f$ can be extended to a Riemann integrable function on the unit torus $\mathbf{S}$, and assume that the atomic part subordinated to $\tilde{\mathbf{Z}}$ of the measure associated to $f$ is 0 (i.e. $\mu\left\{(m+T \mathbf{Z})_{T \in \mathbf{N}}\right\}=0$ $\forall m \in \mathbf{N})$. Then $f=0$ almost everywhere.

Proof. Let $\mu=\left(u_{i}^{T}\right)$. For $j \in \mathbf{N}$ one has

$$
u_{j}^{T}=\frac{1}{T} \sum_{z \in G_{T}} \hat{\mu}(z) z^{j}=\frac{1}{T} \sum_{h=0}^{T-1} \hat{\mu}\left(e^{2 \pi i h / T}\right) e^{2 \pi i h j / T} .
$$


Therefore

$$
u_{j}^{T}=\frac{1}{T} \sum_{h=0}^{T-1} f\left(e^{2 \pi i h / T}\right) e^{2 \pi i h j / T} .
$$

If we denote also by $f$ the extension to $\mathbf{S}$, then the right-hand side is the Riemann sum associated to the integral $\int_{0}^{1} f(t) e^{2 \pi i j t} d t$ so the assumption in the statement of the proposition means that all the Fourier coefficients of $f$ are zero, whence $f$ is equal to zero almost everywhere. (Recall that Theorem $\mathrm{C}$ proves that $u_{j}^{T}$ converges to $\mu\left\{(j+T \mathbf{Z})_{T \in \mathbf{N}}\right\}$ as $T \rightarrow \infty$.)

Proposition 2.3. Let $f$ be a multiplier. Assume that $f$ can be extended to a function on $\mathbf{S}$ such that it is Riemann integrable on some subinterval $I$. If the atomic part subordinated to $\tilde{\mathbf{Z}}$ of the measure associated to $f$ is 0 , then $f$ is equal to zero almost everywhere on $I$.

Proof. Assume $f \neq 0$ a.e. on $I$. Let $g$ be a $C^{\infty}$ function on $\mathbf{S}$ such that $g f$ is Riemann integrable and different from 0 a.e. Denote also by $g$ the restriction of this function to $G$, and take its Fourier inverse $\check{g} \in \mathrm{sp}^{\prime}$ with $\hat{g}=g$. If $g\left(e^{i t}\right)=$ $\sum_{n=-\infty}^{\infty} c_{n} e^{i n t}$ is the Fourier expansion of $g$, then $\check{g}$ is the functional given by $\left(x_{n}\right) \rightarrow \sum_{n=-\infty}^{\infty} c_{n} x_{n}$, since $\check{g} * x=\left(\sum_{n=-\infty}^{\infty} c_{n} S^{n}\right) x$; therefore one has

$$
\hat{g}(z)=\sum_{n=-\infty}^{\infty} c_{n} z^{n}=g(z) .
$$

It follows that the $T$ th row of the matrix associated to $\check{g}$ is given by

$$
\left(\sum_{k=-\infty}^{\infty} c_{k T}, \sum_{k=-\infty}^{\infty} c_{1+k T}, \ldots, \sum_{k=-\infty}^{\infty} c_{T-1+k T}\right) .
$$

Since the function $g$ is $C^{\infty}$, the sequence $\left(c_{n}\right)$ is a rapidly decreasing sequence, so there exists a constant $M$ with

$$
\left|c_{n}\right| \leq M /|n|^{3}, \quad n \neq 0 .
$$

Moreover, with the exception of $j$ and $j-T$, the indices of the $c_{i}$ 's occurring effectively in the series $\sum_{k=-\infty}^{\infty} c_{j+k T}$ have modulus greater than $T$.

Now, denote by $E\left(\right.$ ) the integral part of real numbers, and by $g_{j}^{T}$ the matrix associated to $\check{g}$. Consider the elements

$$
\left(g_{T-E(T / 2)+1}^{T}, \ldots, g_{0}^{T}, \ldots, g_{E(T / 2)}^{T}\right)=\left(b_{-E(T / 2)+1}^{T}, \ldots, b_{0}^{T}, \ldots, b_{E(T / 2)}^{T}\right) .
$$

(This particular disposition will aid us in applying Lebesgue's theorem.) For every one of these elements, say for $b_{h}^{T}$, the lowest modulus of the indices of the summands appearing in its corresponding series is $|h|$. Hence

$$
\begin{gathered}
\left|b_{h}^{T}\right| \leq \sum_{|m| \geq|h|}\left|c_{n}\right| \leq 2 M \sum_{m=|h|}^{\infty} \frac{1}{m^{3}} \quad \text { for } h \neq 0, \\
\left|b_{0}^{T}\right| \leq \sum\left|c_{n}\right|=k
\end{gathered}
$$

Thus the sequence

$$
\left(\ldots, 0, \ldots, 0, b_{-E(T / 2)+1}^{T}, \ldots, b_{0}^{T}, \ldots, b_{E(T / 2)}^{T}, 0, \ldots\right)
$$


is dominated in the index $h$ by the absolutely summable sequence

$$
2 M \sum_{m=|h|}^{\infty}\left(1 / m^{3}\right)
$$

and in $h=0$ by $k$.

On the other hand, one can write

$$
(\check{g} * \mu)_{j}^{T}=\sum_{h=-E(T / 2)+1}^{E(T / 2)} b_{h}^{T} u_{j-h}^{T}
$$

(if $j-h \leq 0$, then let $u_{j-h}^{T}=u_{T+j-h}^{T}$ ).

Since $u_{j}^{T} \rightarrow 0$ as $T \rightarrow \infty$ for all $j$, then $(\check{g} * \mu)_{j}^{T} \rightarrow 0$ as $T \rightarrow \infty$ by the dominated convergence theorem. Moreover, $(\check{g} * \mu)^{\widehat{ }}=g f$ has a Riemann integrable extension to $\mathbf{S}$, so by the previous lemma one concludes that $g f=0$ a.e., which is a contradiction.

ProOF OF THEOREM E. Let $\mu$ be the measure such that $\hat{\mu}=f$. Then $\mu$ takes the form $\nu+\lambda$, where $\nu$ is an atomic measure concentrated in $\tilde{\mathbf{Z}}$ and $\lambda$ is singular to $\mu$. The action of $\nu$ on sp is of the type $x \rightarrow \sum a_{n} x_{n}$, hence $\hat{\nu}(z)=\sum a_{n} z^{n}$ $\left(\sum\left|a_{n}\right|<\infty\right)$, which is obviously Riemann integrable. Since $f$ can be extended to a continuous function on a subinterval $I$ of $G$, the function $\hat{\lambda}$ is Riemann integrable on this subinterval, and by Proposition 2.3 one has $\hat{\lambda}=0$ on $I$. The theorem follows from this fact.

As a particular case, we can study phase-shift filters, namely multipliers of modulus one. Provided they can be extended to a Riemann integrable function, they always are finite Blaschke products:

PROOF OF THEOREM F. Since $f(z)=\sum a_{n} z^{n}, \sum\left|a_{n}\right|<\infty$, the function $f$ is holomorphic on the unit disk $D$ and continuous on $\bar{D}$; therefore it has finitely many zeros $a_{1}, \ldots, a_{p}$ (repeated according to their multiplicity). The Blaschke product

$$
B(z)=\prod_{j=1}^{p} \frac{z-a_{j}}{1-\bar{a}_{j} z}
$$

verifies $|B(z)|=1$ if $|z|=1$. Hence the function $f / B$ is holomorphic on $D$, continuous on $\bar{D}$ and has no zeros, so it is a constant function.

\section{REFERENCES}

1. Luigi Amerio and Giovanni Prouse, Almost periodic functions, Reinhold, New York, 1971.

2. Edwin Hewitt and Kenneth A. Ross, Abstract harmonic analysis. I, Academic Press, New York, 1963.

3. Ronald Larsen, An introduction to the theory of multipliers, Springer-Verlag, New York, 1971.

4. Manuel Valdivia, Topics in locally convex spaces, North-Holland, Amsterdam, 1982.

5. M. E. Munroe, Measure and integration, Addison-Wesley, Reading, Mass., 1971.

6. Walter Rudin, Fourier analysis on groups, Interscience, New York, 1962.

Departamento de Teoría de Funciones, Universidad de Valladolid, 47005 VALLADOLID, SPAIN 